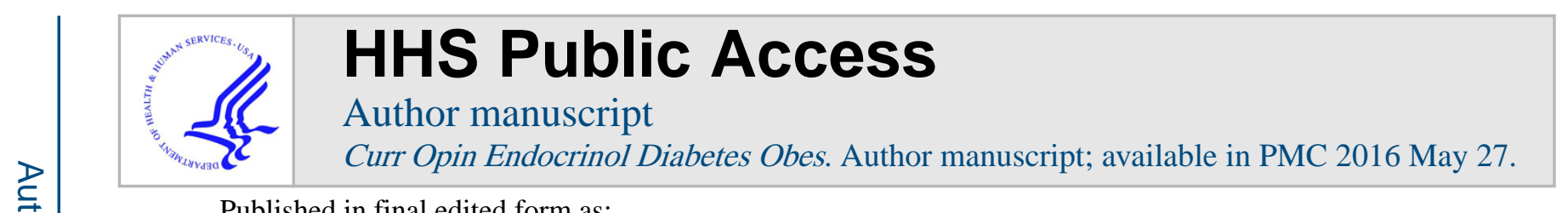

Published in final edited form as:

Curr Opin Endocrinol Diabetes Obes. 2015 December ; 22(6): 483-489. doi:10.1097/MED.

0000000000000206.

\title{
Update on Primary Ovarian Insufficiency
}

\author{
Meghan Hewlett, M.S., M.P.H. ${ }^{a}$ and Shruthi Mahalingaiah, M.D., M.S. ${ }^{a}$ \\ aDepartment of Obstetrics and Gynecology, Boston Medical Center and Boston University, \\ Boston, MA
}

\section{Abstract}

Purpose of Review-Despite an incidence of one percent among women under the age of forty, primary ovarian insufficiency (POI) is still poorly understood. As the variable etiology and presentation of $\mathrm{POI}$ complicate its management, a standard regimen for treatment remains to be established. However, emerging research has provided new insight on current mainstays of treatment as well as novel management approaches and therapeutic interventions.

Recent findings-Recent clinical trials in women with POI indicate that the widely-used regimen of transdermal estradiol and medroxyprogesterone acetate restores bone mineral density (BMD) to a level equal to women with normal ovarian function. Further research verifies that compounded bioidentical hormones and androgen supplementation are inadequate in treating POI and lowering risk for long-term sequelae. Additionally, assessing changes in bone turnover markers may be useful for monitoring BMD. Alternative therapies such as acupuncture, DHEA, and buproprion may be effective in treating the effects of estrogen deficiency at some level, but require further investigation.

Summary-Recent updates show promise in improving management methods and reducing risk of long-term sequelae. Additional research that expands upon the most current literature is critical in order to achieve an evidence-based standard of best practice.

\section{Keywords}

Primary ovarian insufficiency; premature menopause; premature ovarian failure; ovarian dysfunction; estrogen deficiency

\section{Introduction}

Primary ovarian insufficiency (POI), interchangeably termed "premature menopause" or "premature ovarian failure", is defined as the acquisition of hypergonadotropic hypogonadism in women before the age of 40 [1]. The incidence of spontaneous POI is estimated at 1 in 250 for women under 35 years of age and 1 in 100 for women under 40

Full contact for corresponding author (including phone and email): Shruthi Mahalingaiah, M.D., M.S., Department of Obstetrics and Gynecology, Boston Medical Center/Boston University, 85 East Concord St., $6^{\text {th }}$ Floor, Boston, MA 02118, Phone: 617-414-7305, ; Email: shruthi@bu.edu

Conflicts of Interest

None

Any funding disclosures: No disclosures to report 
years of age [2]. The classical presentation of POI includes symptoms associated with estrogen deficiency (i.e. vaginal dryness and hot flashes), menstrual irregularity (oligomenorrhea or amenorrhea), elevated serum gonadotropins, low serum estradiol, and impaired fertility. As women with POI are at an increased risk for a number of chronic sequelae including cardiovascular disease and osteoporosis, early diagnosis and management are critical to prevent the adverse effects of estrogen deficiency. However, as the variable etiology and presentation of POI may complicate its management; a standard regimen for treatment remains to be established. The purpose of this review is to discuss updates on the management of POI.

\section{Management}

As previously described, $\mathrm{POI}$ is associated with a number of potential long-term sequelae including infertility, neurocognitive disorders, and an increased mortality risk. Additionally, women diagnosed with POI, in comparison to controls, have a higher reported incidence of psychological stress, anxiety, depression as well as reduced sexual and general well-being [3]. Given the complexity of this condition, a wide variety of treatment regimens have been recommended; however, none have provided enough evidence to be established as best practice. A multi-dimensional approach to POI management that includes medical treatment, psychosocial support, and preventative care may address the limitations encountered in current practice. Etiological considerations are also important in the management of POI as the cause is variable. Therefore, determining the cause POI for each case following diagnosis is invaluable for selecting the most appropriate and effective method of treatment.

\section{Hormonal Replacement Therapy}

In the absence of contraindications, hormonal replacement therapy (HRT) is strongly advised for not only the treatment of menopausal-related symptoms, but to also reduce the risk of long-term sequelae and neurocognitive function [4]. Previous literature has compared the efficacy of standard sex steroid replacement (sSSR) with physiological sex steroid replacement (pSSR) in managing POI [5-9]. By definition, sSSR comprises treatment regimens made up of synthetic hormonal derivatives, primarily oral ethinylestradiol or combined oral contraceptive pills (COCPs), and were initially utilized for managing symptoms in post-menopausal women. pSSR is characterized as a regimen that includes transdermal estradiol and vaginal progesterone supplementation, and its administration is designed to replicate the natural changes in hormonal levels observed in women with normal ovarian function.

Recent findings indicate that pSSR may be more effective than sSSR in managing POI. sSSR regimens have been shown to treat menopausal symptoms, especially but not limited to vasomotor symptoms and vaginal atrophy; however, they may be inadequate in maintaining skeletal and cardiovascular health as well as improving fertility[10]. In a recent trial comparing improvements in uterine and endometrial parameters between SSSR and pSSR regimens among women with POI, usage of pSSR resulted in a greater mean endometrial thickness compared to sSSR after three months of treatment $(4.8 \mathrm{~mm}$ vs. 3.0 $\mathrm{mm}$, respectively; $\mathrm{p}=0.002)$, thereby indicating a greater improvement in endometrial 
functional response [11]. This study also reported a relatively greater increase in uterine volume for the pSSR regimen compared to sSSR, however, this increase was not statistically significant $(\mathrm{p}=0.070)$. However, this lack of significance may be explained by the etiological make up of the study group as the levels of improvement in uterine parameters associated with pSSR may depend on POI etiology [8]. Therefore, as uterine, endometrial and hypothalamic-pituitary-gonadal functioning must be optimized in order to increase the probability of successful fertility treatment [9], POI patients desiring fertility preservation or pregnancy may benefit from a pSSR regimen. Further research is needed, however, to determine optimal administration protocols for improved success with donor egg therapy. Another recent clinical trial that compared the effect of combined estrogen/progestin therapy in POI patients on bone mineral density (BMD) with controls with normal ovarian function reported that transdermal estradiol and medroxyprogesterone acetate (MPA) (used in pSSR and SSSR, respectively) after three years of treatment maintained BMD in the femoral neck and lumbar spine as well as controls, and restored BMD in the same areas to levels seen in women with normal ovarian function [12]. As the administered hormonal dosing schedule of transdermal estradiol and MPA in this trial is the same regimen most widely used in clinical practice, these findings should help to advocate for prompt treatment and patient compliance.

Preserving Neurocognitive Function-Neurocognitive decline has been reported in the setting of estrogen deficiency [13-16]. A recent population-based cohort study of menopausal women reported that menopause at or before the age of 40 due to premature bilateral ovariectomy or POI was associated with decreased verbal fluency (OR 1.56, 95\% CI $1.12-1.87, \mathrm{P}=0.004)$ and visual memory (OR 1.39, 95\% CI 1.09-1.77, $\mathrm{P}=0.007)$ in later life compared to natural menopause after age 40 [17]. After seven years of follow-up, both surgical and non-surgical menopause occurring before the age of 40 was associated with a $35 \%$ increased risk for significant reductions in psychomotor speed and global cognitive function; however, no significant associations between surgical status groups and these risks were observed. Additionally, HRT did not counteract the negative cognitive effects when taken during premature menopause; however, it may potentially benefit visual memory. As such, timely diagnosis and treatment of POI should continue to be greatly stressed as a means to prevent the detrimental neurocognitive effects of estrogen deficiency that are observed later in life.

Androgens in POI-Androgen supplementation, particularly testosterone, and its usage in improving general and sexual well-being has been investigated [18] but research recommending its usage is controversial. Due to lacking evidence on its safety for long-term usage as well as efficacy, it is not recommended for women with POI. Additionally, no FDAapproved products specifically designed for women with POI have been developed. In a recent study investigating the effects of physiologic testosterone supplementation $(150 \mu \mathrm{g}$ patch) added to an estrogen/progestin regimen among women with POI, no improvements in quality of life, self-esteem, or mood symptoms were observed after 12 months of treatment relative to controls only receiving estrogen/progestin replacement [19]. Based on these findings, there does not appear to be an androgen-related deficiency in the setting of POI. Additionally, while testosterone has been shown to improve BMD in androgen-deficient 
men, $150 \mu \mathrm{g}$ transdermal testosterone added to estrogen/progestin has not shown any additional benefit in maintaining or restoring BMD in women with POI compared to estrogen/progestin alone [12].

Compounded Bioidentical Hormones-In the wake of findings reported by the Women's Health Initiative stating an increased risk of breast cancer and stroke among menopausal women taking HRT, the usage of compounded bioidentical hormone $(\mathrm{CBH})$ greatly increased due to its claims of being a more natural alternative treatment with fewer risks [20] and more effective due to its customized preparation of bioidentical hormones for individual patients. Common formulations of CBHs include Biest (prepared at a ratio of $20 \%$ estradiol and $80 \%$ estriol by weight) and Triest (prepared at a ratio of $10 \%$ estradiol, $10 \%$ estrone, and $80 \%$ estriol by weight) [21]. Compounded preparations are not regulated by the U.S. Food and Drug Administration (FDA), and their legitimacy as bioidentical hormones are not supported by the Endocrine Society or the FDA. Results from a recent four-arm clinical trial of post-menopausal women investigating the pharmacokinetics and therapeutic efficacy of $\mathrm{CBH}(2.0,2.5$, or $3.0 \mathrm{mg}$ of Biest in combination with a $100 \mathrm{mg}$ compounded capsule of progesterone and a placebo patch) with a conventional, FDAapproved regimen $(50 \mu \mathrm{g}$ transdermal estradiol patch with a $100 \mathrm{mg}$ micronized progesterone capsule and a placebo cream) reported greater fluctuations in mean steady state serum estradiol levels with CBH $(11.8 \mathrm{pg} / \mathrm{mL}, 17.8 \mathrm{pg} / \mathrm{mL}$, and $32.2 \mathrm{pg} / \mathrm{mL}$ for the $2.0 \mathrm{mg}$, $2.5 \mathrm{mg}$, and $3.0 \mathrm{mg}$ Biest arms, respectively) compared to the conventional regimen (44.8 $\mathrm{pg} / \mathrm{mL}$ ) [22]. Mean steady state estradiol levels differed significantly between the conventional regimen and the $2.5 \mathrm{mg}$ Biest arm, which is the most common dosage of Biest prescribed $(\mathrm{p}<0.05)$. Additionally, none of the three Biest doses reached the minimum serum estradiol level of $40 \mathrm{pg} / \mathrm{mL}$ recommended for protection against postmenopausal bone loss [23]. Such findings highlight potential inadequacies of $\mathrm{CBH}$ in not only alleviating menopausal symptoms, but also maintaining bone and cardiovascular health and preventing chronic sequelae in women with POI. According to a recent survey, approximately 1 to 2.5 million women aged 40 years or older are estimated to be using CBH therapy in the United States with $86 \%$ reporting that they are not aware that their therapy was not evaluated or approved by the FDA, and 27\% reporting uncertainty as to whether their therapy was customized to their hormone levels [24]. Due to the lack of regulation and the most recently reported research, providers should prioritize patient counseling to inform women diagnosed with POI who are considering CBH therapy about its risks.

DHEA-Dehydroepiandrosterone (DHEA) has been proposed as an alternative hormonal therapeutic for improvement in ovarian function. In a recent clinical trial investigating the effects of DHEA on women with POI through an assessment of ovarian response markers, DHEA significantly increased antral follicle count after 16 weeks of treatment, but did not significantly affect AMH or FSH levels [25]. Nonetheless, DHEA supplementation shows promise in managing POI and warrants further clinical investigation through randomized controlled trials with longer follow-up durations for a more comprehensive assessment of its efficacy. 


\section{Fertility Treatment Options}

Donor Oocytes-Though techniques in fertility preservation for women with primary ovarian insufficiency are still being developed, cryopreserved donor oocyte with in-vitro fertilization should be considered. Historically, fresh donor egg IVF cycles have resulted in high (88\%) cumulative pregnancy rate after 4 cycles [26]. With the development of vitrification, a fast-freeze technique, use of cryopreserved donor oocytes is an option in addition to fresh ovum donation. Rates of post-thaw fertilization, pregnancy, and live birth rates are comparable to fresh donor cycles [27], though no studies have been published on the outcomes in women with premature ovarian insufficiency.

Fertility Preservation-While it remains an investigational method of fertility preservation, ovarian tissue cryopreservation using vitrification with subsequent autotransplantation may be useful in young, particularly pre-pubertal POI patients, which allows for immature oocyte preservation in early ovarian follicles. Thus, a population of mature oocytes could be generated from the early follicles should they successfully develop into pre-ovulatory follicles following transplantation. In a recent publication, 9 out of 24 women with POI exhibiting residual follicles presented with successful follicle growth after graft transplantation beneath Fallopian tubes [28]. Subsequent IVF and embryo transfer in four of these patients resulted in three pregnancies, of which two resulted in successful deliveries. While the authors report that further research on determining the most optimal auto-grafting locations along with detecting residual follicle count in POI patients, cryopreservation of one ovary through this method may serve as a means of early fertility preservation in young patients thereby allowing them to explore non-invasive fertility procedures before undergoing auto-transplantation.

Autoimmune Endocrinopathy Screening-Of the autoimmune disorders, POI is most commonly associated with thyroiditis and autoimmune polyendocrine syndrome, which can include hypothyroidism, adrenal insufficiency, hypoparathyroidism, and type I diabetes mellitus [29]. To screen for asymptomatic autoimmune adrenal insufficiency, serum antiadrenal and anti-21 hydroxylase antibodies should be measured in all women once they are diagnosed with POI and have a confirmed normal karyotype. Furthermore, an anti-21 hydroxylase antibodies may also so serve to diagnose autoimmune oophoritis. However, in rare cases, 21-hydroxlase antibodies may conflict with a positive anti-adrenal antibody test by showing a negative result due to the presence of antibodies to other steroidogenic enzymes such as 17-hyroxylase [30]. While screening for adrenal antibodies is available [29], a reliable, standardized screening test for ovarian antibodies has yet to be established, and thus the prevalence of autoimmune oophoritis in the absence of adrenal autoimmunity among women with POI is currently unknown. Additionally, normal karyotypic young women diagnosed with POI have an increased risk of autoimmune hypothyroidism and thus assessments of thyroid stimulating hormone (TSH), free thyroxine (T4), and anti-thyroidperoxidase and anti-thyroglobulin antibodies should be taken [31].

In regard to clinical evaluation, timely identification of abnormal immunological activity in the setting of suspected POI could help to confirm a diagnosis of autoimmune POI and theoretically restore ovarian function with early administration of appropriate 
immunosuppressive treatment regimen. Previous research has also investigated inflammatory markers and their utility as a potential biomarker for POI. A recent study comparing neutrophile to lymphocyte ratio (NLR) between POI cases and normal controls found that NLR was significantly lower in POI cases $(\mathrm{p}<0.001)$ and that an NLR ratio less than or equal to 1.5 was an independent risk factor for POI [32]. While increases in NLR are associated with increases in the severity of inflammatory pathology, prior literature has proposed that lower NLRs are associated with the presence of autoimmunity or chronic inflammation. Further investigations examining the diagnostic accuracy of NLR are warranted. NLR could potentially serve to identify immunological abnormalities, thereby narrowing the field of potential etiologies for a particular case of POI. A more accurate determination of the cause of POI will help to establish more specialized treatment regimens thereby potentially producing more effective outcomes.

Bone Turnover Markers-Age- and menopausal status-related changes in bone turnover markers have been observed in POI, and they may thus serve as an assessment for routine BMD monitoring in POI management. Commonly measured bone turnover markers include bone-specific alkaline phosphatase (bALP) and procollagen type I C-peptide (PICP), both bone formation markers, and cross-linked N-telopeptides of type I collagen (NTX), a marker of bone resorption. In a recent study examining the impact of POI on bone turnover markers, all bone turnover markers, BALP, NTX, and PICP were significantly negatively correlated in women with POI [33]. When stratified into two age subgroups using the age of thirty, the age at approximate peak BMD, as a cutoff, POI patients younger than 30 years of age had significantly lower lumbar t-scores compared to POI patients aged 30 years or older (mean \pm standard deviation (SD): $-1.84 \pm 1.47$ vs. $-1.06 \pm 0.93$; $p<0.021$ ). The authors also reported that POI patients under 30 years of age had higher bALP (mean (U/l) \pm SD: 48.99 \pm 42.16 vs. $23.76 \pm 10.08 ; \mathrm{p}=0.004$ ) and NTX levels (mean (nmol) \pm SD: $58.80 \pm 21.32$ vs. $41.10 \pm 11.37 ; \mathrm{p}<0.001)$. Estrogen deficiency may thus have a greater impact on BMD decline in younger women with POI, particularly if they have not yet reached peak BMD, as previous research shows that increases in bone turnover markers are associated with an increased risk of adverse bone health including bone fracture [34]. However, reference intervals for bone turnover markers have not been fully established due to variations between studies from potentially non-equivalent study populations based on differing inclusion and exclusion factors [35-39]. Further efforts to establish standardized assessment methods and reference values are needed in order to effectively utilize bone turnover markers in POI management.

Alternative Management Strategies-In conjunction with HRT, mainstay lifestyle management approaches and regimens to maintain BMD include weight-bearing exercises, promotion of increased calcium and vitamin D through diet and/or supplementation, and cessation of alcohol and tobacco [40]. For women with POI who are not taking HRT due to contraindications or personal decision, it is well known that these alternative management strategies are insufficient in preventing the decline in BMD [40]. Additionally, none of these lifestyle recommendations can help treat menopausal symptoms. As such, women under this protocol should receive routine BMD every 6-12 months [40]. Currently, there is limited evidence to support the efficacy of non-hormonal treatment options such as complementary 
therapies, selective serotonin re-uptake inhibitors (SSRIs), Venlafaxine, or Gabapentin. In a study of 31 POI patients investigating the therapeutic efficacy of acupuncture, three months of acupuncture administered three times a week resulted in significant reductions in average serum FSH $(\mathrm{p}<0.01)$ and LH $(\mathrm{p}<0.01)$, as well as a significant increase in average serum estradiol $(p<0.01)$ [41]. Significant reductions in Self-Rated Anxiety Score following treatment were also reported $(\mathrm{p}<0.001)$. However, as the study group comprised of only 31 patients, such results may not fully represent the response of women with POI to acupuncture. Based on current research, these alternative regimens may be helpful in alleviating menopausal symptoms, but their ability to reduce the long-term effects of estrogen deficiency is controversial and thus they are not recommended for routine management.

Psychopharmacological Management-Treatment of depression is complicated in POI as selective serotonin reuptake inhibitors (SSRIs) have historically been shown to be associated with decreases in lower lumbar spine and hip BMDs after five years of use [42, 43]. Additionally, SSRI's and tricyclic antidepressants are associated with dose-dependent increases in bone fracture risk [44]. However, such effects on bone metabolism have not been observed in the setting of estrogen deficiency, indicating that skeletal effects of antidepressants may be influenced by sex steroid levels [45]. Additionally, in a very recent study investigating the effects of bupropion, an atypical antidepressant, on bone metabolism in ovariectomized rats, treatment was osteoprotective and dose-dependently inhibited increases in bone turnover markers as well as inflammatory cytokines [45]. Treatment also resulted in a complete remission of bone loss after 6 weeks of treatment at both low $(30 \mathrm{mg} / \mathrm{kg} *$ day $)$ and high doses $(60 \mathrm{mg} / \mathrm{kg} *$ day). While bupropion does not directly treat estrogen deficiency, it shows promise for POI patients diagnosed with depression in preventing osteoporotic sequelae.

\section{Conclusion}

Despite increases in emerging research, POI remains poorly understood due to its variable etiology and complex morbidity. Establishing a diagnosis and its potential etiology as quickly as possible is critical to prevent the precipitous increases in risk for chronic sequelae due to estrogen deficiency including infertility, cardiovascular disease, osteoporosis, and neurocognitive decline. Additionally, effective management necessitates a multidisciplinary approach that incorporates HRT, fertility management, maintenance of BMD, diet and exercise, as well as routine screening, applicable psychopharmacological treatment, and psychosocial support. Continued research must be prioritized for the much-needed establishment of evidence-based diagnosis and treatment guidelines.

\section{Acknowledgments}

\section{Financial Support and Sponsorship}

S.M. is supported by the Reproductive Scientist Development Program from the NIH/NICHD Grant K12 HD000849. 


\section{References}

Papers of particular interest, published within the annual period of review, (18 months/20122013) have been highlighted as:

- of special interest

•• of outstanding interest

1. Nelson LM. Clinical practice. Primary ovarian insufficiency. N Engl J Med. 2009; 360(6):606-14. [PubMed: 19196677]

2. Coulam CB, Adamson SC, Annegers JF. Incidence of premature ovarian failure. Obstet Gynecol. 1986; 67(4):604-6. [PubMed: 3960433]

3*. Kovanci E, Schutt AK. Premature ovarian failure: clinical presentation and treatment. Obstetrics and gynecology clinics of North America. 2015; 42(1):153-61. An up-to-date review of the potential etiologies, clinical picture, diagnostic workup, and management of primary ovarian insufficiency. This review uses available evidence to provide clinical recommendations within these areas of focus and discusses knowledge gaps in the literature. [PubMed: 25681846]

4*. Scott EL, Zhang QG, Vadlamudi RK, Brann DW. Premature menopause and risk of neurological disease: basic mechanisms and clinical implications. Molecular and cellular endocrinology. 2014; 389(1-2):2-6. This review provides a detailed discussion of the research examining the detrimental effects of premature menopause on neurological functioning. This article also discusses the available and needed research ascertaining the optimal time to initiate estrogen supplementation in order to maintain neurological health. [PubMed: 24462786]

5. Sidney S, Cheetham TC, Connell FA, Ouellet-Hellstrom R, Graham DJ, Davis D, et al. Recent combined hormonal contraceptives (CHCs) and the risk of thromboembolism and other cardiovascular events in new users. Contraception. 2013; 87(1):93-100. [PubMed: 23083525]

6. Chataigneau T, Zerr M, Chataigneau M, Hudlett F, Hirn C, Pernot F, et al. Chronic treatment with progesterone but not medroxyprogesterone acetate restores the endothelial control of vascular tone in the mesenteric artery of ovariectomized rats. Menopause. 2004; 11(3):255-63. [PubMed: 15167304]

7. Crofton PM, Evans N, Bath LE, Warner P, Whitehead TJ, Critchley HO, et al. Physiological versus standard sex steroid replacement in young women with premature ovarian failure: effects on bone mass acquisition and turnover. Clinical endocrinology. 2010; 73(6):707-14. [PubMed: 20738314]

8. Biljan MM, Taylor CT, Matijevic R, Jones SV, Garden AS, Fraser WD, et al. Exaggerated effects of progestogen on uterine artery pulsatility index in Turner's syndrome patients receiving hormone replacement therapy. Fertil Steril. 1995; 64(6):1104-8. [PubMed: 7589660]

9. Remohi J, Ardiles G, Garcia-Velasco JA, Gaitan P, Simon C, Pellicer A. Endometrial thickness and serum oestradiol concentrations as predictors of outcome in oocyte donation. Hum Reprod. 1997; 12(10):2271-6. [PubMed: 9402294]

10*. Sassarini J, Lumsden MA, Critchley HO. Sex hormone replacement in ovarian failure - new treatment concepts. Best practice \& research Clinical endocrinology \& metabolism. 2015; 29(1): 105-14. This article compares the efficacy of standard sex steroid replacement with physiological sex steroid replacement in treating menopausal symptoms, improving fertility, and preventing long-term sequelae in women with premature ovarian failure. This review concentrates on the progress made in the literature assessing the risk/benefit ratio of both regimens. [PubMed: 25617176]

11. O'Donnell RL, Warner P, Lee RJ, Walker J, Bath LE, Kelnar CJ, et al. Physiological sex steroid replacement in premature ovarian failure: randomized crossover trial of effect on uterine volume, endometrial thickness and blood flow, compared with a standard regimen. Hum Reprod. 2012; 27(4):1130-8. [PubMed: 22343553]

12*. Popat VB, Calis KA, Kalantaridou SN, Vanderhoof VH, Koziol D, Troendle JF, et al. Bone mineral density in young women with primary ovarian insufficiency: results of a three-year randomized controlled trial of physiological transdermal estradiol and testosterone replacement. J Clin Endocrinol Metab. 2014; 99(9):3418-26. These findings highlight the effectiveness of 
estrogen and progestin therapy on osteoporotic health after long-term treatment in women with primary ovarian insufficiency. Thus there is more evidence to support the administration of a combined usage of physiological and standard sex steroid replacement therapy as best practice in treating primary ovarian insufficiency. [PubMed: 24905063]

13. Shuster LT, Rhodes DJ, Gostout BS, Grossardt BR, Rocca WA. Premature menopause or early menopause: long-term health consequences. Maturitas. 2010; 65(2):161-6. [PubMed: 19733988]

14. Vearncombe KJ, Pachana NA. Is cognitive functioning detrimentally affected after early, induced menopause? Menopause. 2009; 16(1):188-98. [PubMed: 18724262]

15. Henderson VW, Sherwin BB. Surgical versus natural menopause: cognitive issues. Menopause. 2007; 14(3 Pt 2):572-9. [PubMed: 17476147]

16. Rocca WA, Bower JH, Maraganore DM, Ahlskog JE, Grossardt BR, de Andrade M, et al. Increased risk of cognitive impairment or dementia in women who underwent oophorectomy before menopause. Neurology. 2007; 69(11):1074-83. [PubMed: 17761551]

17*. Ryan J, Scali J, Carriere I, Amieva H, Rouaud O, Berr C, et al. Impact of a premature menopause on cognitive function in later life. BJOG. 2014; 121(13):1729-39. One of the few studies to assess the influence of primary ovarian insufficiency on cognitive decline. This study further supports previous literature that primary ovarian insufficiency negatively affects cognitive function later in life and also shows that there is no significant improvement in cognitive function with estrogen replacement therapy. [PubMed: 24802975]

18. Floter A, Nathorst-Boos J, Carlstrom K, von Schoultz B. Addition of testosterone to estrogen replacement therapy in oophorectomized women: effects on sexuality and well-being. Climacteric. 2002; 5(4):357-65. [PubMed: 12626215]

19*. Guerrieri GM, Martinez PE, Klug SP, Haq NA, Vanderhoof VH, Koziol DE, et al. Effects of physiologic testosterone therapy on quality of life, self-esteem, and mood in women with primary ovarian insufficiency. Menopause. 2014; 21(9):952-61. This article is one of the very few studies to assess the effect of testosterone supplementation when added to hormonal replacement therapy in treating psychosocial symptoms in primary ovarian insufficiency. Of note, there does not appear to beneficially nor detrimentally affect psychosocial outcomes. [PubMed: 24473536]

20. Fishman JR, Flatt MA, Settersten RA Jr. Bioidentical hormones, menopausal women, and the lure of the "natural" in U.S. anti-aging medicine. Soc Sci Med. 2015; 132:79-87. [PubMed: 25795991]

21. Boothby LA, Doering PL, Kipersztok S. Bioidentical hormone therapy: a review. Menopause. 2004; 11(3):356-67. [PubMed: 15167316]

22. Sood R, Warndahl RA, Schroeder DR, Singh RJ, Rhodes DJ, Wahner-Roedler D, et al. Bioidentical compounded hormones: a pharmacokinetic evaluation in a randomized clinical trial. Maturitas. 2013; 74(4):375-82. [PubMed: 23384975]

23*. Davis R, Batur P, Thacker HL. Risks and effectiveness of compounded bioidentical hormone therapy: a case series. J Womens Health (Larchmt). 2014; 23(8):642-8. This review comprehensively examines the efficacy of compounded bioidentical hormones in treating menopausal symptoms, which are an increasingly prevalent alternative to standard HRT regimens. The authors compare the current evidence and reaffirm that research is lacking on their effectiveness. [PubMed: 25111856]

24. Pinkerton JV, Santoro N. Compounded bioidentical hormone therapy: identifying use trends and knowledge gaps among US women. Menopause. 2015

25. Yeung TW, Li RH, Lee VC, Ho PC, Ng EH. A randomized double-blinded placebo-controlled trial on the effect of dehydroepiandrosterone for 16 weeks on ovarian response markers in women with primary ovarian insufficiency. J Clin Endocrinol Metab. 2013; 98(1):380-8. [PubMed: 23144466]

26. Paulson RJ, Hatch IE, Lobo RA, Sauer MV. Cumulative conception and live birth rates after oocyte donation: implications regarding endometrial receptivity. Hum Reprod. 1997; 12(4):835-9. [PubMed: 9159452]

27. Cobo A, Meseguer M, Remohi J, Pellicer A. Use of cryo-banked oocytes in an ovum donation programme: a prospective, randomized, controlled, clinical trial. Hum Reprod. 2010; 25(9):223946. [PubMed: 20591872] 
28. Suzuki N, Yoshioka N, Takae S, Sugishita Y, Tamura M, Hashimoto S, et al. Successful fertility preservation following ovarian tissue vitrification in patients with primary ovarian insufficiency. Hum Reprod. 2015; 30(3):608-15. [PubMed: 25567618]

29. Rebar, R. Evaluation of Amenorrhea, Anovulation, and Abnormal Bleeding. In: De Groot, LJ.; Beck-Peccoz, P.; Chrousos, G.; Dungan, K.; Grossman, A.; Hershman, JM., et al., editors. Endotext. South Dartmouth (MA): 2000.

30. American College of O, Gynecologists Committee on G. ACOG Committee Opinion No. 469: Carrier screening for fragile X syndrome. Obstet Gynecol. 2010; 116(4):1008-10. [PubMed: 20859177]

31. Kim TJ, Anasti JN, Flack MR, Kimzey LM, Defensor RA, Nelson LM. Routine endocrine screening for patients with karyotypically normal spontaneous premature ovarian failure. Obstet Gynecol. 1997; 89(5 Pt 1):777-9. [PubMed: 9166320]

32. Yldrm G, Tokmak A, Kokanal MK, Sarkaya E, Zungun C, Inal HA, et al. Association between some inflammatory markers and primary ovarian insufficiency. Menopause. 2015

33*. Kurtoglu-Aksoy N, Akhan SE, Bastu E, Gungor-Ugurlucan F, Telci A, Iyibozkurt AC, et al. Implications of premature ovarian failure on bone turnover markers and bone mineral density. Clinical and experimental obstetrics \& gynecology. 2014; 41(2):149-53. This is one of the few studies to compare bone turnover markers and bone mineral density in women with primary ovarian insufficiency using the estimated age of peak bone mass as a benchmark for analysis. * Results of this study show that similar correlations between bone mineral density and bone turnover marker levels are similar to those seen in post-menopausal women. [PubMed: 24779240]

34. Naylor K, Eastell R. Bone turnover markers: use in osteoporosis. Nat Rev Rheumatol. 2012; 8(7): 379-89. [PubMed: 22664836]

35. Eastell R, Garnero P, Audebert C, Cahall DL. Reference intervals of bone turnover markers in healthy premenopausal women: results from a cross-sectional European study. Bone. 2012; 50(5): 1141-7. [PubMed: 22348982]

36. Li M, Li Y, Deng W, Zhang Z, Deng Z, Hu Y, et al. Chinese bone turnover marker study: reference ranges for $\mathrm{C}$-terminal telopeptide of type I collagen and procollagen I $\mathrm{N}$-terminal peptide by age and gender. PLoS One. 2014; 9(8):e103841. [PubMed: 25117452]

37. de Papp AE, Bone HG, Caulfield MP, Kagan R, Buinewicz A, Chen E, et al. A cross-sectional study of bone turnover markers in healthy premenopausal women. Bone. 2007; 40(5):1222-30. [PubMed: 17331821]

38. Glover SJ, Garnero P, Naylor K, Rogers A, Eastell R. Establishing a reference range for bone turnover markers in young, healthy women. Bone. 2008; 42(4):623-30. [PubMed: 18289953]

39. Glover SJ, Gall M, Schoenborn-Kellenberger O, Wagener M, Garnero P, Boonen S, et al. Establishing a Reference Interval for Bone Turnover Markers in 637 Healthy, Young, Premenopausal Women From the United Kingdom, France, Belgium, and the United States. Journal of Bone and Mineral Research. 2009; 24(3):389-97. [PubMed: 18665786]

40*. Cox L, Liu JH. Primary ovarian insufficiency: an update. International journal of women's health. $2014 ; 6: 235-43$. A comprehensive review discussing the current mainstays of diagnosis and management of primary ovarian insufficiency. This provides a sufficient background to inform future efforts.

41. Chen Y, Fang Y, Yang J, Wang F, Wang Y, Yang L. Effect of acupuncture on premature ovarian failure: a pilot study. Evidence-based complementary and alternative medicine : eCAM. 2014; 2014:718675. [PubMed: 24711856]

42. Patterson-Buckendahl P, Kvetnansky R, Fukuhara K, Cizza G, Cann C. Regulation of plasma osteocalcin by corticosterone and norepinephrine during restraint stress. Bone. 1995; 17(5):46772. [PubMed: 8579958]

43. Patterson-Buckendahl P, Rusnak M, Fukuhara K, Kvetnansky R. Repeated immobilization stress reduces rat vertebral bone growth and osteocalcin. Am J Physiol Regul Integr Comp Physiol. 2001; 280(1):R79-86. [PubMed: 11124137]

44. Cizza G, Primma S, Csako G. Depression as a risk factor for osteoporosis. Trends in endocrinology and metabolism: TEM. 2009; 20(8):367-73. [PubMed: 19747841] 
45**. Abuohashish HM, Ahmed MM, Al-Rejaie SS, Eltahir KE. The antidepressant bupropion exerts alleviating properties in an ovariectomized osteoporotic rat model. Acta Pharmacol Sin. 2015; 36(2):209-20. A novel investigation into the effects of buproprion, an atypical antidepressant, on bone mineral density in the setting of estrogen deficiency. While this is an animal study, these findings suggest a potentially effective pharmacotherapeutic method of treating depression in primary ovarian insufficiency without significant detriment to osteoporotic health. [PubMed: 25544359] 


\section{Key Points}

- Women with primary ovarian insufficiency seeking fertility may benefit from physiological sex steroid replacement; however, further research is needed to determine optimal administration protocols for improved success with donor egg therapy.

- As transdermal estradiol and medroxyprogesterone acetate has been shown to maintain restore bone mineral density in women with primary ovarian insufficiency, educating patients prescribed the regimen on the benefits of longterm treatment compliance is recommended.

- Due to the lack of regulation and the most recently reported research regarding compounded bioidentical hormones, providers should prioritize patient counseling to inform women diagnosed with primary ovarian insufficiency who are considering the therapy about its risks.

- Further efforts to establish standardized assessment methods and reference values are needed in order to effectively utilize bone turnover markers for monitoring bone mineral density over the course of managing patients diagnosed with primary ovarian insufficiency.

- While bupropion does not directly treat estrogen deficiency, it shows promise for primary ovarian insufficiency patients diagnosed with depression in preventing osteoporotic sequelae.

- Though autologous ovarian tissue harvesting, cryopreservation and subsequent in-vitro maturation and fertilization techniques are experimental, cryopreserved donor oocyte should be considered. 